\title{
Research Paper: The Association of Depressive Symptoms With Plasma C-Reactive Protein in Patients With Major Depressive Disorder Under Treatment
}

\author{
Azher Nema Mohammed Al-Agam ${ }^{1}$ (D), Ammar Waheeb Obeiad² (D, Mahir Abdulkadhum Khudhair Alzughaibi² (D), Hayder Abdul-Amir M. \\ Al-Hindy ${ }^{3^{*}}$ (1), Amer Fadhil Alhaideri ${ }^{4}$ (i)
}

1. Department of Psychiatry, Merjan Teaching Hospital, Babil Health Directorate, Babylon, Iraq

2. Merjan Teaching Hospital, Babil Health Directorate, Babylon, Iraq.

3. Department of Pharmacology and Toxicology, School of Pharmacy, University of Babylon, Hilla City, Babylon, Iraq

4. Department of Medicine, School of Medicine, Karbala University, Karbala, Iraq.

\begin{tabular}{|c|c|}
\hline $\begin{array}{l}\text { Use your device to scan } \\
\text { and read the article online }\end{array}$ & citation Al-Agam A, Obeiad A, Alzughaibi M, Makki H, Alhaideri AF. The Association of Depressive Symptoms With \\
\hline 口ifirita: & $\begin{array}{l}\text { Plasma C-Reactive Protein in Patients With Major Depressive Disorder Under Treatment. Iranian Rehabilitation Journal. 2021; } \\
\text { 19(4):425-432. http://dx.doi.org/10.32598/irj.19.4.1619.1 }\end{array}$ \\
\hline 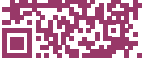 & doi'http://dx.doi.org/10.32598/irj.19.4.1619.1 \\
\hline
\end{tabular}

Article info:

Received: 02 Oct 2021

Accepted: 06 Oct 2021

Available Online: 01 Dec 2021

Keywords:

Major Depressive Disorder (MDD), The 9-Item Patient Health Questionnaire (PHQ-9), C-Reactive Protein (CRP), Depression, Low-grade inflammation

\section{ABSTRACT}

Objectives: This study evaluates the relationship between plasma high sensitive c-reactive protein (HSCRP) in patients with Major Depressive Disorder (MDD) under therapy.

Methods: This cross-sectional study included 90 patients with MDD that had been diagnosed previously to confirm their matching «DSM-5 criteria for MDD version 7.0.2,» employing the «mini-international neuropsychiatric interview.» Also, they were on antidepressants prescriptions for at least 4 months. The criteria of MDD were based on the self-administered inquiry forms for evaluating depression severity, comprising the 9-item Patient Health Questionnaire (PHQ-9) depression module. A venous blood sample was collected from all participants for White Blood Cells (WBCs) counts and HSCRP assays. Besides their BMI calculations, SPSS v. 23, was used for all statistical tests.

Results: HSCRP mean serum levels were within normal ranges among MDD patients. The Mean \pm SD age of the MDD patients was $39.5 \pm 0.9$ years, and most of them were obese; their Mean \pm SD BMI was $32.9 \pm 15.8 \mathrm{~kg} / \mathrm{m}^{2}$. The mean WBCs count of the participants was within the normal ranges. The ratio of male/female participants in this study was 1.64:1. There was a nonsignificant difference between the sexes in all study parameters: no significant variations in the distribution of HSCRP levels according to the scores of depression severity. There was no significant variation in the distribution of WBCs counts according to the severity of depressive thoughts. A receiver operating characteristic curve (when tested for the diagnostic ability of HSCRP) revealed poor predictability to distinguish those with severe MDD from those with no or mild depressive thoughts: area under the curve $=0.484$, sensitivity $=0.52$, specificity $=0.46$, and $\mathrm{P}>0.05$.

Discussion: The outcomes of our study highlighted the importance of low-grade inflammation as a risk factor of the onset or even worsening of depression in patients with MDD. This finding is reflected by a significant difference in the mean levels of serum HSCRP between those having mild and severe PHQ-9 scores. However, the mean serum levels of HSCRP were not correlated with the severity of depressive symptoms.

\section{* Corresponding Author:}

Hayder Abdul-Amir M. Al-Hindy, MD.

Address: Department of Pharmacology and Toxicology, School of Pharmacy, University of Babylon, Hilla City, Babylon, Iraq

Tel: +96 (47) 708020200

E-mail:phar.hayder.abdul@uobabylon.edu.iq 


\section{Highlights}

- Although MDD is not a pure inflammatory disorder, inflammation vigorously contributes to the severity of depression.

- There is a significant difference in the mean levels of HSCRP between those having mild and those with mild to severe depression based on the 9-item PHQ-9 depression module scores.

- Low-grade inflammation is a significant risk factor for the onset or exacerbation of depression in patients with MDD.

- Further studies should explore the mechanisms involved in the association of inflammation and MDD, which may improve understanding of these conditions and design more targeted therapies.

\section{Plain Language Summary}

As a prevailing disorder, depression can result in substantial burdens and debilities worldwide. Of many emerging recent risk factors, inflammation had a documented contribution to several human diseases. C-reactive protein (CRP) is a known biomarker of systemic body inflammation. Our goal in this study was to assess the role of low-grade inflammation in patients with depression. The study revealed that the depressive patients exhibited higher levels of CRP in their blood, though not correlated with the severity of the symptoms. Meanwhile, there is a significant difference between those having mild and those with moderate to severe depressive scores regarding the mean levels of CRP. These findings suggest the significant role of low-grade inflammation in depressive patients that may predict the onset or even aggravation of the depression symptoms. This observation is essential in future studies to develop more targeted therapies that could help patients with depression.

\section{Introduction}

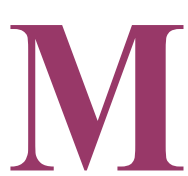

ajor Depressive Disorder (MDD) is a prevailing disorder that leads to substantial burden and debilities worldwide [1]. It may primarily increase death owing to suicide or secondarily by deteriorating prospects of chronic disorders like Coronary Vascular Disease (CVD) [2]. The wellrecognized association between symptoms of MDD and CVD has been doubled the risk of evolving ischemic heart disease [3]. Even though the customary risk factors of CVD tend to gather in depressive subjects due to unhealthy living standards (e.g., poor nutrition, absence of exercise), these ill manners do not sufficiently responsible for the influence of depression on CVD. A potential systematic link between MDD and CVD is low-grade inflammation. Based on the available evidence, depression and CVD are mutual illnesses that frequently arise together. The evidence suggests their planned coincidence and the risk factor of depression by its neuroendocrine pathways induces the pathogenesis and evolution of coronary arteriosclerosis and later heart disease $[4,5]$. Although MDD lacks a pure inflammatory pathology, inflammation shows a massive contribution [6] that may predict the onset of depression [7].
Alternatively, higher serum levels of High Sensitive C-Reactive Protein (HSCRP) are a biomarker of a lowgrade inflammation $[8,9]$ associated with the occurrence and prognosis of CVD [10-15]. Consequently, both depression and HSCRP are related to CVD events. Nonetheless, the exact mechanism of their association is still unclear. Several epidemiological studies have described a relationship between depressive symptoms and higher HSCRP in the blood [16-18]. Some academics propose a probable psychoneuroimmunology association between negative affection (depression, rage, nervousness, poor welfare), inflammatory biomarkers, and the evolution of cardiovascular events [19]. The study aimed to evaluate the relationship between plasma HSCRP levels with depressive symptoms, in people with MDD under therapy.

\section{Materials and Methods}

In this cross-sectional study, the patients with MDD were selected by the psychiatrists at Merjan Teaching Hospital, including the author. The study included 90 adults with previously diagnosed MDD. To confirm their matching "DSM-5 criteria for MDD version 7.0.2" , we employed the mini-international neuropsychiatric interview [20]. They were on antidepressants prescriptions for at least 4-months. MDD patients had enrolled from those attending psychiatric consultation clinics of 
Table 1. Basal demographic features of the studied participants

\begin{tabular}{cccrc}
\hline Characteristics & CRP & Age & BMI & 32.9 \\
\hline Mean & 3.1 & 39.45 & 3.2 & 3.0 \\
Median & 0.06 & 37.0 & 15.1 & 2.2 \\
Std. Deviation & 0.9 & 15.8 & 26.0 & 4.0 \\
Minimum & 0.0 & 12.0 & 41.0 \\
Maximum & 0.0 & 82.0 & 14.0 \\
\hline
\end{tabular}

CRP: C-Reactive Protein; BMI: Body Mass Index; WBC: White Blood Cell.

Mranian Rehabilitation Journa

Merjan Teaching Hospital between April and June 2021, throughout their follow-up appointments. The criteria of MDD were based on the self-administered inquiry forms obtainable for evaluating depression severity, comprising the 9-item Patient Health Questionnaire (PHQ-9) depression module [21]. MDD applicants identify the incidence of every thought that arose throughout the previous week (minimum: 1=not at all to maximum: 4=nearly every day), where upper scores represent higher thought incidence. The overall score is the sum of inquiries 1-30 and labels the incidence of thoughts.

The exclusion criteria included any neurological illnesses like epilepsy, injured or degenerative brain illnesses, drug addicts, and steroid users within the past two months. It is well known that MDD must not be established or omitted only based on a PHQ-9 scoring. A score of PHQ-9 $\geq 10$ is $88 \%$ sensitive and $88 \%$ specific for MDD [22]. Based on this module, the depressed subjects were classified into five groups (scores): (0-4) no depression, (5-9) mild, (10-14) moderate depression, (15-20) moderately severe, and (21-27) severe depression. In the end, a decisive diagnosis was made based on how well the subject understood the inquiry form and other related data from the depressive patient [21].

Fresh venous samples from the participants were collected, centrifuged, and stored for further hematological and biochemical assays. The sampling times were matched among all the applicants. Leukocytes counts were measured based on the available classical methods. Measurements of HSCRP were achieved following the company instructions of "Calbiotech ${ }^{\circledR}$ Netherland, using High Sensitivity C-reactive Protein ELISA kit." Participants' body weight, length, and body mass index were accurately measured.

The data were collected, processed, and examined using SPSS v. 23, USA-IBM. Variations in demographic features were assessed using the t-test for continuous variables and the Chi-square test for categorical variables. The Pearson correlations were applied to examine the associations between the parameters.

\section{Results}

The applicants' characteristics are available in Table 1. The HSCRP mean serum levels were within the normal range among MDD patients. The Mean \pm SD age of the MDD patients was $39.5 \pm 0.9$ years, and most patients were obese; their Mean \pm SD BMI was $32.9 \pm 15.8 \mathrm{~kg} / \mathrm{m}^{2}$. The mean WBC counts of the participants were within the normal range.

The ratio of a male/female in this study was 1.64:1. There was a non-significant difference between males and females in all study parameters (Table 2). There were no significant differences in the mean concentrations of serum HSCRP according to the types of antidepressants used by patients with MDD (results not shown) (Table 3). Likewise, no significant variations were seen in the distribution of HSCRP levels regarding the severity of depression scores (Table 4). There was no significant variation in the distribution of WBCs counts in terms of severity of depressive thoughts (Table 5).

Figure 1 displays a significant difference in the mean levels of serum HSCRP between those with low and those with high PHQ-9 scores. Those who were severely symptomatic showed higher levels of HSCRP in their sera. Receiver operation characteristics, when tested for the diagnostic ability of HSCRP, revealed poor predictability to distinguish those with severe MDD from those with no or mild depressive thoughts: area under the curve $(\mathrm{AUC})=0.484$, sensitivity $=0.52$, specificity $=0.46$, and $\mathrm{P}$ $>0.05$ (Figure 2). 
Table 2. Sex differences of the study parameters in the participants

\begin{tabular}{ccccc}
\hline Characteristics & Sex & Mean & Std. Error Mean & Significance \\
\hline HSCRP $(\mathrm{mg} / \mathrm{mL})$ & F & 10.2 & 1.4 & $>0.05$ \\
Age $(\mathrm{y})$ & M & 7.5 & 1.3 & $>0.05$ \\
F & 41.1 & 2.1 & $>0.05$ \\
BMI $\left(\mathrm{kg} / \mathrm{m}^{2}\right)$ & M & 36.9 & 2.7 & $>0.05$ \\
WBC $(1 \times 103 / \mu \mathrm{L})$ & 32.6 & 4.4 & \\
& M & 32.9 & 0.4 & 0.4 \\
\hline
\end{tabular}

HSCRP: high sensitive C-reactive protein; BMI: Body Mass Index; WBC, white blood cell.

Iranian Rehabilitation Journa

\section{Discussion}

The current study explored the associations of inflammatory biomarkers with patients with MDD among the Iraqi people. The research revealed no direct association between depression and inflammation; however, the mean serum levels of HSCRP were significantly higher in depressive patients with higher PHQ-9 scores than those with lower scores. Our finding is hard to rationalize with a growing body of evidence in current years that has proposed a close association between low-grade inflammation, cytokines levels, and MDD. One of the foremost attentions of this field is the contribution of the immune system in mental wellbeing and psychiatric illnesses $[6,7,23]$. The macrophage concept of depression assumes that the secretion of proinflammatory cytokines by triggered macrophages subsidizes the onset or exacerbation of MDD [24]. Furthermore, in the latest large meta-analysis, a wide range of variations in immune response have been defined in depressive patients, such as greater concentrations of tumor necrosis factoralpha, interleukin (IL)-6, IL-13, IL-18, IL-12, IL-1RA, and sTNFR2, as well as a fall in the cytokine interferongamma [25].
On the other hand, a suppressed inflammatory response has also been reported in depressed subjects [26]. Patients with MDD have a more leukocyte count and CD4+/ CD8+ratio, besides less natural killer (NK) cell number with weakened $\mathrm{T}$ and NK cell activity as described by a previous meta-analysis [26]. There are few studies revealing inconsistent results of immune stimulation and or suppression in MDD. Indeed, both may ensue in the same subject, like suppression of NK and regulatory Tcell action along with monocytes activation [27].

Meanwhile, a recent study published results similar to our findings and revealed that depression is related to inflammation only in individuals with exact characteristics. The researchers concluded that the associations between MDD, inflammatory response, and covariates were probably highly multivariable and multifactorial that deserve further analysis [28]. A recent review has described a bidirectional association between MDD and inflammation [29]. The multicausal association comprises the likelihood of reverse causality, where depression is not a result, instead the reason for greater inflammatory levels. Besides, the authors found that relations between depressive symptoms and inflammatory biomarkers were weakened after adjusting covariate; BMI and gender constantly exhibited strong associations with inflammatory

Table 3. Mean serum HSCRP levels with respect to WBCs, BMI, and age, which was significant only with WBCs

\begin{tabular}{lcccc}
\hline & \multicolumn{1}{c}{ Correlation } & WBCs & BMI & Age \\
\hline \multirow{H}{*}{$\operatorname{HSCRP}(\mathrm{mg} / \mathrm{mL})$} & Pearson Correlation & 0.42 & 0.139 & 0.11 \\
& Sig. (2-tailed) & 0.000 & 0.19 & 0.3 \\
\hline
\end{tabular}


Table 4. Distribution of HSCRP levels in terms of the depression severity

\begin{tabular}{cccc}
\hline Severity of Depression & Number & Mean \pm SD & P \\
\hline No depression & 0 & 0 & $3.0 \pm 2.4$ \\
Mild depression & 6 & $10.3 \pm 8.7$ & $>0.05$ \\
Moderate depression & 25 & $9.2 \pm 9.7$ \\
Moderate-Severe & 59 & 0 \\
Severe & 0 & \\
\hline
\end{tabular}

HSCRP: High Sensitive C-Reactive Protein.

Mranian Rehabilitation Journa

markers [28]. In the same channel, a highly significant rise of CRP levels was not detected among all MDD patients, but only among those with atypical type or suicidal thoughts, as reported by Egyptian scholars recently [30].

In a systematic review and focused meta-analysis on the association between CRP and MDD, a minor association $(r=0.05)$ was found after adjustment of age, gender, body weight, health conditions, and treatments or psychosocial issues. The influence size was extremely attenuated and not significant in surveys of high-quality scoring. Furthermore, the authors define several recommendations for upcoming studies to consider, like sample assortment and analyzing measures, data filtering, and statistical approaches, and control parameters to evaluate [31].

Numerous factors can alter the inflammatory response and confound the levels of CRP in the blood among MDD subjects. The role of gender on HSCRP levels may be more intricate in the perspective of depressive thoughts. Several studies on CRP and MDD have found conflicting results; some reported raised CRP values in men, but not women [32], and other studies revealed the opposite [33]. Medicines, like statins [34], antihypertensive medication [35], non-steroidal anti-inflammatory drugs [36], and even antidepressants [37], are linked with both CRP and MDD. Some substances like alcohol or caffeine consumption and nicotine (smoking) are associated with CRP values dysregulation [38]. The alternative confounding issue is obesity, in which evidence proposes that the association between CRP and BMI is possibly determined mostly by obesity [39]. Of note, obesity in our study was common. Socioeconomic status has been consistently and inversely associated with serum levels of HSCRP, often independent of demographic, biological, or behavioral issues [31].

Based on the results mentioned above, the theory that MDD is linked with the inflammatory immune system may be an effective target for therapy. Additional works should emphasize the exact pathways involved in the association of inflammation and MDD, which may help us improve our understanding of these conditions and design more targeted therapies.

\section{Conclusion}

There was a significant difference in the mean levels of serum HSCRP between those having low and those with high PHQ-9 scores. Those who were severely symptomatic revealed higher levels of HSCRP in their sera. No significant correlation was found between the mean serum levels of HSCRP with symptoms of MDD.

Table 5. Difference in the distribution of WBCs count in terms the severity of depressive thoughts

\begin{tabular}{cccc}
\hline Variable & Severity of Depression & Mean \pm SD & $P$ \\
\hline \multirow{2}{*}{ WBCs count } & Mild & $7.740 \pm 1.8746$ & 0.12 \\
& Moderate- severe & $8.565 \pm 2.4142$ & \\
\hline
\end{tabular}

WBC: white blood cell.

Iranian Riehabilitation \ourna 


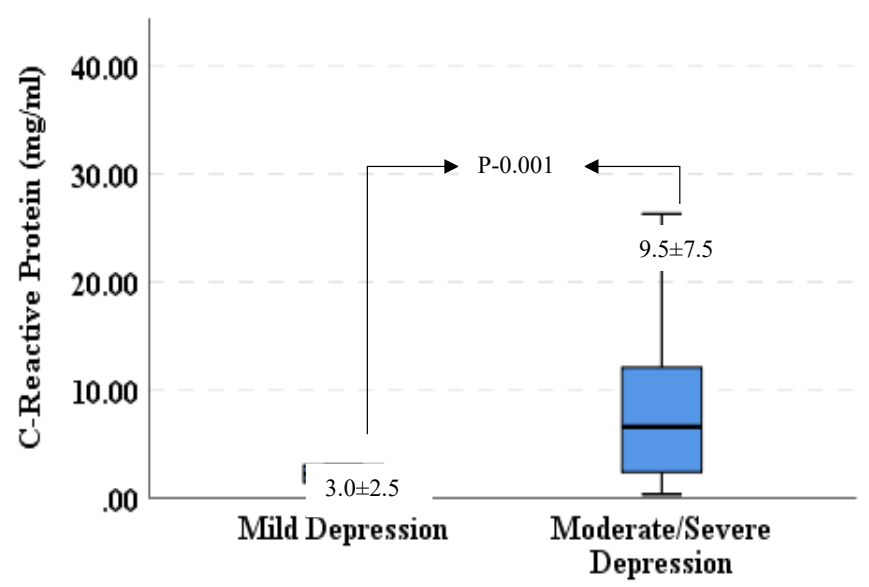

Figure 1. Boxplot distribution of HSCRP in terms of depression severity based on PHQ-9 scores

Пranian Rehabilitation \ournal HSCRP: high sensitive C-reactive protein; PHQ-9: the 9-item patient health questionnaire.

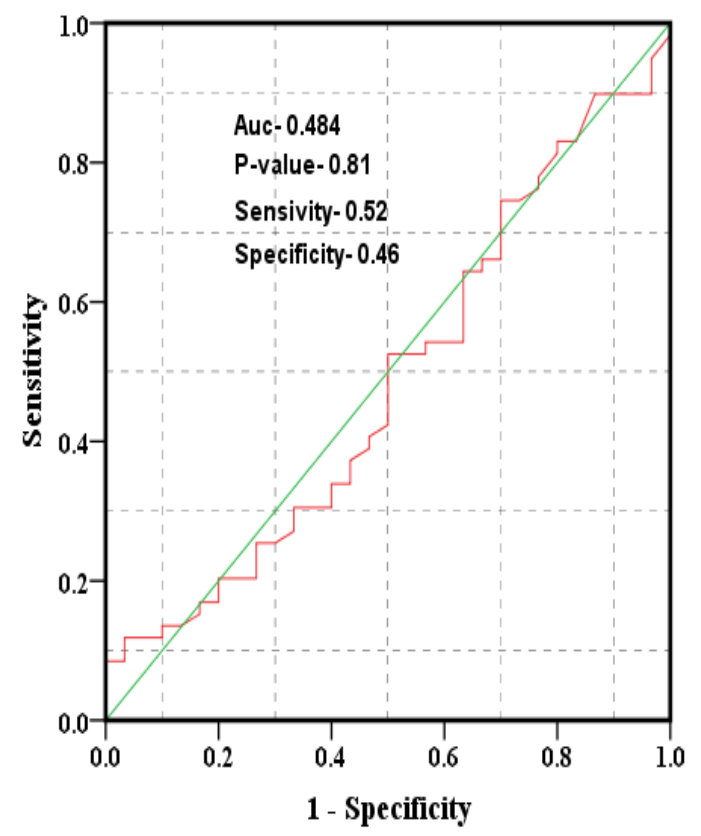

Iranian Rehabilitation \ournal

Figure 2. ROC-Curve Diagnostic Ability of HSCRP to Distinguish Those With Severe MDD From Those With no or Mild Depressive Thoughts

HSCRP: high sensitive C-reactive protein; MDD: major depressive disorder; ROC-curve: receiver operating characteristic curve.

\section{Ethical Considerations}

\section{Compliance with ethical guidelines}

This study and its protocol were approved by the authority of the health institution at Merjan Teaching Hospital (Code: MH., EC: 12-8-2020, 110772). Also, we applied the American Psychological Association's ethical principles. The involved applicants (or their relatives) provided a written informed agreement. The study did not include any clinical therapeutic trial. The whole research was conducted according to the Helsinki Declaration.

Funding

This research did not receive any grant from funding agencies in the public, commercial, or non-profit sectors. 


\section{Authors' contributions}

Conceptualization: Hayder Abdul-Amir M. Al-Hindy, Amer Fadhil Alhaideri; Methodology: Amer Fadhil Alhaideri, Azher Nema Mohammed Al-Agam; Investigation: Ammar Waheeb Obeiad; Writing - original draft: Hayder Abdul-Amir M. Al-Hindy, Mahir Abdulkadhum Khudhair Alzughaibi; Writing - review \& editing: All authors; Supervision: Hayder Abdul-Amir M. Al-Hindy.

\section{Conflict of interest}

The authors declared no conflict of interest.

\section{References}

[1] Moussavi S, Chatterii S, Verdes E, Tandon A, Patel V, Ustun B. Depression, chronic diseases, and decrements in health: Results from the World Health Surveys. The Lancet. 2007; 370(9590):851-8. [DOI:10.1016/S0140-6736(07)61415-9]

[2] Van der Kooy K, van Hout H, Marwijk H, Marten H, Stehouwer C, Beekman A. Depression and the risk for cardiovascular diseases: Systematic review and meta analysis. In ternational Journal of Geriatric Psychiatry. 2007; 22(7):613-26. [DOI:10.1002/gps.1723] [PMID]

[3] Rosanski A BJ, Davidson KW, Saab PG, Kubzansky L. The epidemiology, pathophysiology, and management of psychosocial risk factors in cardiac practice: The emerging field of behavioral cardiology. Journal of the American College of Cardiology. 2005; 45(5):637-51. [DOI:10.1016/j.jacc.2004.12.005] [PMID]

[4] Sundquist J, Li X, Johansson SE, Sundquist K. Depression as a predictor of hospitalization due to coronary heart disease. American Journal of Preventive Medicine. 2005; 29(5):428-33. [DOI:10.1016/j.amepre.2005.08.002] [PMID]

[5] Vieweg WV, Julius DA, Fernandez A, Wulsin LR, Mohanty PK, Beatty-Brooks M, et al. Treatment of depression in patients with coronary heart disease. The American Journal of Medicine. 2006; 119(7):567-73. [DOI:10.1016/j.amjmed.2006.02.037] [PMID]

[6] Lee CH, Giuliani F. The Role of Inflammation in Depression and Fatigue. Frontiers in Immunology. 2019; 10:1696. [DOI:10.3389/fimmu.2019.01696] [PMID] [PMCID]

[7] Ernst M, Brähler E, Otten D, Werner AM, Tibubos AN Reiner I, et al. Inflammation predicts new onset of depression in men, but not in women within a prospective, representative community cohort. Scientific Reports. 2021; 11(1):2271. [DOI:10.1038/s41598-021-81927-9] [PMID] [PMCID]

[8] Al-Hindy HA, Al-Athari AJ, Mousa MJ, Hameed SJ, Obeed $\mathrm{SH}$. The utility of serum IL-1 $\beta$ and CRP together with fractional exhaled Nitric Oxide in the diagnosis of asthma in adults. NeuroQuantology. 2021; 19(8):119-24. [DOI:10.14704/ nq.2021.19.8.NQ21122]
[9] Jawad AL-Daami Q, Bash HS, Naji GH, AL-Hindy HA. High-sensitivity C-Reactive protein assessment in bronchial asthma: Impact of exhaled Nitric Oxide and body mass index. Systematic Reviews in Pharmacy. 2020; 11(3):705-11. [DOI:10.31838/srp.2020.3.96]

[10] Al-Saad RZ, Shaker AK, Dleikh FS, Al-Hindy HA. Is there any association between highly sensitive C-Reactive protein and dental-status in ischemic heart diseases? A Comparative Study. Biochemical and Cellular Archives. 2020; 20(2):6069-75. https://connectjournals.com/toc2. php? abstract $=3256502 \mathrm{H} \_6069 \mathrm{~A}$.pdf\&\&bookmark=CJ033216\&\&issue_id $=\& \& y a e r=2020$

[11] Mohammed SM, Hasan AS, Al-Hindy HA, Mousa MJ. CReactive protein is associated with the severity of periodontal disease? An observational study among acute myocardial infarction patients. Systematic Reviews in Pharmacy. 2020; 11(10):252-7. [DOI:10.31838/srp.2020.10.41]

[12] Al-Mumin A, Al-Hindy HA, Mousa MJ. Combined assessments of multi-panel biomarkers for diagnostic performance in coronary artery disease: Case-control analysis. Systematic Reviews in Pharmacy. 2020; 11(6):665-71. https:/ / www.sysrevpharm.org/abstract/combined-assessments-of-multipanel-biomarkers-for-diagnostic-performance-in-coronaryartery-disease-casecontrol-analysis-65948.html\#cite

[13] Shaker AK, Al-Saad R, Jasim R, Al-Hindy HA. Biochemical significance of cystatin-C and high-sensitive CRP in patients with acute coronary syndrome; any clinical correlation with diagnosis and ejection fraction. Systematic Reviews in Pharmacy. 2020; 11(3):301-8. https://www.sysrevpharm.org/ abstract/biochemical-significance-of-cystatinc-and-highsensitive-crp-in-patients-with-acute-coronary-syndrome-anyclinical-correl-65730.html

[14] Abdul_Husseein HK, Dleikh FS, Al-Aaraji AJ, Al-Hindy HA, Mousa MJ. Biochemical causal-effect of circulatory uric acid, and HSCRP and their diagnostic correlation in admitted patients with ischemic heart diseases. Journal of Cardiovascular Disease Research. 2020; 11(2):25-31. [DOI:10.31838/ jcdr.2020.11.02.06]

[15] Al-hindi HA, Mousa MJ, Al-Kashwan TA, Sudan A, Abdul-Razzaq SA. On admission levels of high sensitive C-Reactive protein as A biomarker in acute myocardial infarction: A case-control study. Indian Journal of Public Health Research \& Development. 2019; 10(4):1480-4. [DOI:10.5958/09765506.2019.00924.0]

[16] Danner M, Kasl SV, Abramson JL, Vaccarino V. Association between depression and elevated C-reactive protein. Psychosomatic Medicine. 2003; 65(3):347-56. [DOI:10.1097/01. PSY.0000041542.29808.01] [PMID]

[17] Fond G, Micoulaud-Franchi JA, Faugere M, Boyer L, FagetAgius C, Lançon C, et al. Abnormal C-reactive protein blood levels as a specific biomarker of major depression and nonremission under antidepressants in schizophrenia. Progress in Neuro-Psychopharmacology and Biological Psychiatry. 2020; 97:109800. [DOI:10.1016/j.pnpbp.2019.109800] [PMID]

[18] Pitharouli MC, Hagenaars SP, Glanville KP, Coleman JR, Hotopf M, Lewis CM, et al. Elevated C-Reactive protein in patients with depression, independent of genetic, health, and psychosocial factors: Results from the UK Biobank. American Journal of Psychiatry. 2021; 178(6):522-9. [DOI:10.1176/appi. ajp.2020.20060947] [PMID] 
[19] Ma Y, Chiriboga DE, Pagoto SL, Rosal MC, Li W, Merriam PA, et al. Association between depression and C-Reactive protein. Cardiology Research and Practice. 2010; 2011:286509. [DOI:10.4061/2011/286509] [PMCID]

[20] Sheehan DV, Lecrubier Y, Sheehan KH, Amorim P, Janavs J, Weiller E, et al. The Mini-International Neuropsychiatric Interview (M.I.N.I.): The development and validation of a structured diagnostic psychiatric interview for DSM-IV and ICD-10. The Journal of Clinical Psychiatry. 1998; 59(S 20):2233; quiz 4-57. [PMID]

[21] Kroenke K, Spitzer RL, Williams JB. The PHQ-9: Validity of a brief depression severity measure. Journal of General Internal Medicine. 2001; 16(9):606-13. [DOI:10.1046/j.15251497.2001.016009606.x] [PMID] [PMCID]

[22] National Institute of Mental Health (NIMH). Depression. 2016. Retrieved 22 October 2020. https://www.nimh.nih gov/health/publications/depression

[23] Osimo EF, Baxter LJ, Lewis G, Jones PB, Khandaker GM. Prevalence of low-grade inflammation in depression: A systematic review and meta-analysis of CRP levels. Psychological Medicine. 2019; 49(12):1958-70. [DOI:10.1017/ S0033291719001454] [PMID] [PMCID]

[24] Smith RS. The macrophage theory of depression. Medical Hypotheses. 1991; 35(4):298-306. [DOI:10.1016/03069877(91)90272-Z]

[25] Köhler CA, Freitas TH, Maes M, de Andrade NQ, Liu CS, Fernandes BS, et al. Peripheral cytokine and chemokine alterations in depression: A meta-analysis of 82 studies. Acta Psychiatrica Scandinavica. 2017; 135(5):373-87. [DOI:10.1111/ acps.12698] [PMID]

[26] Blume J, Douglas SD, Evans DL. Immune suppression and immune activation in depression. Brain, Behavior, and Immunity. 2011; 25(2):221-9. [DOI:10.1016/j.bbi.2010.10.008] [PMID] [PMCID]

[27] Grosse L, Hoogenboezem T, Ambrée O, Bellingrath S, Jörgens S, de Wit HJ, et al. Deficiencies of the $\mathrm{T}$ and natural killer cell system in major depressive disorder: $\mathrm{T}$ regulatory cell defects are associated with inflammatory monocyte activation. Brain, Behavior, and Immunity. 2016; 54:38-44. [DOI:10.1016/j.bbi.2015.12.003] [PMID]

[28] Fried EI, von Stockert S, Haslbeck JMB, Lamers F, Schoevers RA, Penninx BWJH. Using network analysis to examine links between individual depressive symptoms, inflammatory markers, and covariates. Psychological Medicine. 2020 50(16):2682-90. [DOI:10.1017/S0033291719002770] [PMID]

[29] Beurel E, Toups M, Nemeroff CB. The bidirectional relationship of depression and inflammation: Double trouble. Neuron. 2020; 107(2):234-56. [DOI:10.1016/j.neuron.2020.06.002] [PMID] [PMCID]

[30] Mohamed AE, El-Latif RRA, Youssef AM, Ibrahim AS. Creactive protein and clinical subtypes of major depressive disorder at Zagazig University Hospitals. Middle East Current Psychiatry. 2020; 27(1):35. [DOI:10.1186/s43045-020-00038-9]

[31] Horn SR, Long MM, Nelson BW, Allen NB, Fisher PA, Byrne ML. Replication and reproducibility issues in the relationship between C-reactive protein and depression: A systematic review and focused meta-analysis. Brain, Behavior, and Immunity. 2018; 73:85-114. [DOI:10.1016/j.bbi.2018.06.016] [PMID] [PMCID]
[32] Häfner S, Emeny RT, Lacruz ME, Baumert J, Herder C, Koenig W, et al. Association between social isolation and inflammatory markers in depressed and non-depressed individuals: Results from the MONICA/KORA study. Brain, Behavior, and Immunity. 2011; 25(8):1701-7. [DOI:10.1016/j. bbi.2011.06.017] [PMID]

[33] Nazmi A, Oliveira IO, Victora CG. Correlates of C-reactive protein levels in young adults: A population-based cohort study of 3827 subjects in Brazil. Brazilian Journal of Medical and Biological Research. 2008; 41(5):357-67. [DOI:10.1590/ S0100-879X2008000500003] [PMID]

[34] Prasad K. C-reactive protein (CRP)-lowering agents. Cardiovascular Drug Reviews. 2006; 24(1):33-50. [DOI:10.1111/ j.1527-3466.2006.00033.x] [PMID]

[35] Di Napoli M, Papa F. Angiotensin-converting enzyme inhibitor use is associated with reduced plasma concentration of C-reactive protein in patients with first-ever ischemic stroke. Stroke. 2003; 34(12):2922-9. [DOI:10.1161/01. STR.0000099124.84425.BB] [PMID]

[36] Solheim S, Arnesen H, Eikvar L, Hurlen M, Seljeflot I. Influence of aspirin on inflammatory markers in patients after acute myocardial infarction. The American Journal of Cardiology. 2003; 92(7):843-5. [DOI:10.1016/S0002-9149(03)00897-X]

[37] Hollon SD, Thase ME, Markowitz JC. Treatment and prevention of depression. Psychological Science in the Public Interest: A Journal of the American Psychological Society. 2002; 3(2):39-77. [DOI:10.1111/1529-1006.00008] [PMID]

[38] O'Connor MF, Bower JE, Cho HJ, Creswell JD, Dimitrov S, Hamby ME, et al. To assess, to control, to exclude: Effects of biobehavioral factors on circulating inflammatory markers. Brain, Behavior, and Immunity. 2009; 23(7):887-97. [DOI:10.1016/j.bbi.2009.04.005] [PMID] [PMCID]

[39] Timpson NJ, Nordestgaard BG, Harbord RM, Zacho J, Frayling TM, Tybjærg-Hansen A, et al. C-reactive protein levels and body mass index: Elucidating direction of causation through reciprocal Mendelian randomization. International Journal of Obesity (2005). 2011; 35(2):300-8. [DOI:10.1038/ ijo.2010.137] [PMID] [PMCID] 